\title{
Mechanism of Tetrandrine Against Endometrial Cancer Based on Network Pharmacology
}

\author{
Wenqian Shang' \\ Jing Zhang ${ }^{2}$ \\ Haibo Song ${ }^{2}$ \\ Shunfei Zhu ${ }^{3}$ \\ Aimin Zhang' \\ Yushuang Hua' \\ Shujun $\mathrm{Han}^{4}$ \\ Yan $\mathrm{Fu}^{5}$
}

'Department of Traditional Chinese Medicine, Zibo Maternal and Child Health Hospital, Zibo, Shandong, 255000, People's Republic of China; ${ }^{2}$ Translational Medicine Center, Zibo Maternal and Child Health Hospital, Zibo, Shandong, 255000, People's Republic of China; ${ }^{3}$ Clinical Laboratory, Zibo Maternal and Child Health Hospital, Zibo, Shandong, 255000, People's Republic of China;

${ }^{4}$ Surgical Department, Zhangdian District Hospital of Traditional Chinese Medicine, Zibo, 255000, Shandong, People's Republic of China; ${ }^{5}$ Shandong University of Technology, Zibo, 255000, Shandong, People's Republic of China
Correspondence: Aimin Zhang; Wenqian Shang

Department of Traditional Chinese

Medicine, Zibo Maternal and Child Health Hospital, No. 66 North Tianjin Road,

Zhangdian District, Zibo, Shandong, People's Republic of China

Tel +86 I8505330408; +86 I5053358995

Email purplesunk@163.com;

shanzhongyiwanyi@I26.com
Background: Endometrial cancer (EC) is one of the most common gynaecological malignancies, and its incidence has been rising over the past decade. Tetrandrine, a bisbenzylisoquinoline alkaloid, has been isolated from a vine used in traditional Chinese medicine, Stephania tetrandra. However, the key mechanism of tetrandrine in EC is still unclear.

Purpose: This research was designed to predict the molecular mechanisms of tetrandrine against EC based on network pharmacology and to further verify these predictions by in vitro experiments.

Methods: The potential therapeutic targets of tetrandrine against EC were predicted by using public databases. Afterwards, the protein-protein interaction (PPI) network of the common targets was constructed, and the key gene targets were obtained. Biological function and pathway enrichment analyses were performed by Gene Ontology (GO) and Kyoto Encyclopedia of Genes and Genomes (KEGG) analyses. Furthermore, molecular docking and in vitro experiments were carried out to verify the predictions. The cell counting kit- 8 (CCK-8) assay, Hoechst 33258 staining, flow cytometry analysis, qRT-PCR, Western blot analysis and an immunofluorescence assay were performed.

Results: Our findings identified 111 potential therapeutic targets of tetrandrine against EC. We obtained 7 key gene targets from the PPI network analysis. Furthermore, GO enrichment analysis indicated that these targets were mainly associated with metabolic processes, responses to stimulus, and biological regulation. The KEGG pathway analysis showed that the common targets were mainly distributed in the PI3K/Akt signalling pathway. A potential interaction of tetrandrine with Aktl was revealed by molecular docking. In addition, in vitro experiments showed that tetrandrine significantly inhibited cell proliferation and induced apoptosis in Ishikawa and HEC-1-B cells in dose- and time-dependent manners. The results also revealed that tetrandrine can downregulate the expression of $\mathrm{Bcl}-2$ and upregulate the expression of Bax at the mRNA level. The mRNA levels of Akt were not significantly different in the various tetrandrine $(0,10$ and $20 \mu \mathrm{M})$ groups. However, Western blot analysis demonstrated that the protein expression ratios of $\mathrm{p}$-Akt/Akt decreased at the protein level. The results were further confirmed by immunofluorescence assays.

Conclusion: Based on bioinformatic analysis and experimental verification, our findings demonstrated that tetrandrine exerted tumour-suppressive effects on EC by regulating the PI3K/Akt signalling pathway.

Keywords: endometrial carcinoma, mechanism, network pharmacology, tetrandrine

\section{Introduction}

Endometrial cancer (EC) is one of the most common gynaecological malignancies. According to statistics from the American Cancer Society in 2021, endometrial 
cancer is increasing in both incidence and mortality. ${ }^{1}$ Surgical treatment is the mainstay for initial management of endometrial cancer; chemotherapy and hormone therapy are the main treatments for women with advanced and recurrent disease. ${ }^{2}$ In clinical applications, compared with traditional chemotherapy drugs with obvious adverse effects, traditional Chinese medicine (TCM) offers the advantages of high efficacy and low toxicity. ${ }^{3}$ As an interesting research hotspot, TCM can possibly regulate oncogenes and tumour suppressor genes, epigenetic modifications, microenvironments, and cancer stem cells. $^{4}$ In recent decades, increasing numbers of cancer patients have used TCM as an alternative therapy.

Tetrandrine (Tet) is a bisbenzylisoquinoline (BBI) alkaloid that is extracted from Stephania tetrandra S. Moor, a member of Menispermaceae. ${ }^{5}$ In addition, tetrandrine has a variety of pharmacological activities, including antitumour, anti-inflammatory, immunosuppressant, antiallergic, anticancer, and antioxidant activities. ${ }^{6}$ In recent years, many studies have revealed that tetrandrine has anticancer effects on various tumours, such as cervical cancer, nasopharyngeal cancer, breast cancer, colon cancer, oral cancer and prostate cancer. ${ }^{7-12}$ However, the effect and mechanism of tetrandrine on EC have not been reported thus far.

Network pharmacology approaches differ from conventional drug discovery by addressing the ability of drugs to target numerous proteins or networks that are involved in a disease. ${ }^{13}$ In drug discovery, network pharmacology approaches are increasingly being developed and applied. ${ }^{14}$ Moreover, this approach effectively bridges the gap between modern medicine and TCM. ${ }^{15}$

Hence, we identified the key gene targets and signalling pathways of tetrandrine against EC by using network pharmacology approaches. Afterwards, we validated the predictions by using a series of in vitro assays. All procedures used in this report are shown in Figure 1.

\section{Materials and Methods}

\section{Drug and Disease Targets Identification}

The target proteins of tetrandrine were obtained from the TCMSP ${ }^{16}$, (https://tcmspw.com/tcmsp.php), DrugBank (https://www.drugbank.ca/), and PharMapper ${ }^{17-19}$ (http:// www.lilab-ecust.cn/pharmmapper/). The molecular structures of tetrandrine were obtained from PubChem (https:// pubchem.ncbi.nlm.nih.gov/). The target proteins that we screened were standardized in UniProt (http://www.uni prot.org/). The target genes associated with endometrial

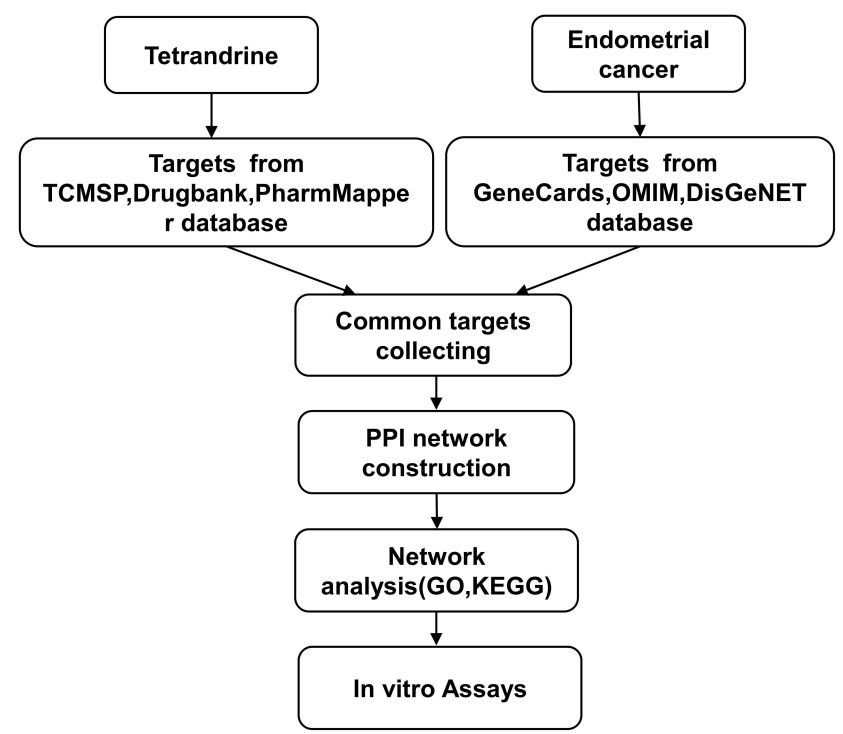

Figure I Workflow for the identification potential mechanism of tetrandrine in treating endometrial cancer.

cancer were acquired from GeneCards (https://www.gene $\underline{\text { cards.org/) }}$ with relevance scores $\geq 10$, and OMIM (https:// omim.org/) and DisGeNET (http://www.disgenet.org/) with disease scores $\geq 0.8$. The common targets of tetrandrine and EC were identified by using Venny 2.1 (http:// bioinfogp.cnb.csic.es/tools/venny/index.html).

\section{Protein-Protein Interaction (PPI) Network Construction}

STRING is an online database for protein-protein interaction networks. The common targets were input into the STRING database (https://string-db.org/) to generate a protein-protein interaction network, with the species limited to "Homo sapiens" and confidence ranges selected as high confidence (score value $>0.9$ ). Afterwards, the PPI network data were input into Cytoscape3.7.2 to analyse and obtain the core genes. In addition, Cytoscape3.7.2 software was used to construct a "tetrandrine-EC-target" network and to screen hub genes.

\section{Gene Function and Pathway Enrichment Analysis}

Gene Ontology (GO) and Kyoto Encyclopedia of Genes and Genomes (KEGG) pathway analyses were performed to analyse the common targets by using WebGestalt (http://www.webgestalt.org/option.php). KEGG pathway enrichment analysis (http://www.genome.jp/kegg/) was employed to obtain a pathway map of the core pathway. 


\section{Molecular Docking Analysis}

The structure of tetrandrine was retrieved from PubChem (https://pubchem.ncbi.nlm.nih.gov/). The Protein Data Bank database was used to retrieve the receptor protein, AKT1 (PDBID: 4EKL). The receptor proteins were dehydrated, and ligands were removed by using PyMOL2.3.4 software. AutoDock tools were used to modify the receptor protein, such as changing the hydrogenation and charge balance. AutoDock Vina 1.1.2 was used to dock the receptor protein with the small molecule ligand. The affinity scores were used to evaluate the binding potential between Akt1 and tetrandrine.

\section{Reagents}

Tetrandrine (purity $\geq 98 \%$ ) was purchased from Aladdin Biochemical Technology Co. (Shanghai, China). Tetrandrine was dissolved in DMSO (Solarbio Science \& Technology Co., Beijing, China) and was then added to the cell culture supernatant in appropriate proportions. FBS and tyrosine were obtained from Gibco (Carlsbad, CA, USA). Cell Counting Kit-8 (CCK-8), Hoechst 33258 and Annexin V-PI apoptosis reagents were purchased from Beyotime (Shanghai, China). The TRIzol reagent and qRT-PCR Kit were purchased from Invitrogen (Carlsbad, CA, USA). A cDNA Synthesis Kit was from Takara (Shiga, Japan). The antibodies against Bax, Bcl-2, Akt, p-Akt, and $\beta$-actin were purchased from Cell Signalling Technology, Inc. (Danvers, MA, USA). The HRP and FITC goat anti-rabbit antibodies were purchased from Wanleibio Co. (Shenyang, China). The relevant primers were synthesized by Rui Biotech Co. (Qingdao, China).

\section{Cell Culture}

The Ishikawa endometrial carcinoma cell line was provided by the Laboratory of Fudan University Affiliated Obstetrics and Gynaecology Hospital. The use of the Ishikawa cell lines was approved by our institutional research ethics committee. The HEC-1-B endometrial carcinoma cell line was obtained from the Shanghai Institute for Biological Sciences. Ishikawa cells were cultured in RPMI-1640 (HyClone, UT, USA), and HEC-1-B cells were cultured in MEM (HyClone, UT, USA), all of which were supplemented with $10 \%$ fetal bovine serum and $1 \%$ penicillin-streptomycin. Both cell lines were cultured under a humidified atmosphere at $37^{\circ} \mathrm{C}$ with $5 \%$ $\mathrm{CO}_{2}$.

\section{Cell Viability Assay}

Cell viabilities were determined by CCK-8 assays. The Ishikawa and HEC-1-B cells were seeded into 96-well plates. Then, both cell lines were treated with different tetrandrine concentrations $(0,5,10,20,40$, and $80 \mu \mathrm{M})$. After 24, 48, and $72 \mathrm{~h}$, a CCK-8 solution was added to each well and then cultured in an incubator for 2 hours. Then, the optical densities were measured at $450 \mathrm{~nm}$ by using a microplate reader (Thermo Scientific, MA, USA).

\section{Hoechst 33258 Staining}

The Ishikawa and HEC-1B cells were added to 12-well plates and incubated with tetrandrine $(0,10$, and $20 \mu \mathrm{M})$ for $24 \mathrm{~h}$. The cultured cells were fixed with $4 \%$ paraformaldehyde for $30 \mathrm{~min}$ and were then washed three times with PBS. Subsequently, the cells were stained with $5 \mu \mathrm{g}$ / $\mathrm{mL}$ Hoechst 33258 for $5 \mathrm{~min}$ in the dark and washed with PBS. All samples were observed and photographed with a fluorescence microscope (Olympus, Tokyo, Japan) at $\times 200$.

\section{Cell Apoptosis Assay}

Both cell lines were seeded into 6-well plates and treated with $0,10 \mu \mathrm{M}$ and $20 \mu \mathrm{M}$ tetrandrine for $24 \mathrm{~h}$. The cells were washed with PBS, digested by trypsin, centrifuged and collected. Thereafter, the collected cells were resuspended in $100 \mu \mathrm{L}$ of PBS and stained in the dark with 5 $\mu \mathrm{L}$ of Annexin $\mathrm{V}$ and $10 \mu \mathrm{L}$ of PI for $15 \mathrm{~min}$. The samples were detected by a FACSCalibur flow cytometer (BD Pharmingen, CA, USA) within $1 \mathrm{~h}$ after staining.

\section{Quantitative Real-Time Polymerase Chain Reaction (qRT-PCR) Analysis}

The Ishikawa and HEC-1B cells were added to 6-well plates and incubated with tetrandrine $(0,10$, and $20 \mu \mathrm{M})$. After $24 \mathrm{~h}$, total RNA was extracted using TRIzol reagent according to the manufacturer's instructions. The total RNA concentrations were determined by using an ultra-micro spectrophotometer (DeNovix DS11, DE, USA). After the quality and concentrations of RNA were determined, cDNA was synthesized with the PrimeScript RT Reagent Kit by following the protocol described by the manufacturer. The mRNA expression levels were quantified using a SYBR Green qPCR Kit on a QuantStudio 3 system (Applied Biosystems, USA). All primers were designed using Primer Premier 5.0 software (Premier Biosoft, www.premierbiosoft.com). The 
primer sequences were designed as follows: Bcl-2: forward: 5'-GTGTGTGGAGAGCGTCAACC-3', reverse: 5'-CAGAGACAGCCAGGGAAATCA-3'; Bax: forward: 5'-GACTGATGTCCCTGTCTCCAG-3', reverse: 5'CTCCCGCCACAAAGATGGTC-3'; and Akt: forward: 5'-CTCTTTCCAGACCCACGACC-3', reverse: 5'- TAA TGTGCCCGTCCTTGTCC-3'; $\beta$-actin: forward: $5^{\prime}$ ACCGCGAGAAGATGACCCAG-3', reverse: 5'GGATAGCACAGCCTGGATAGCAA-3'. The target gene expressions were normalized to the housekeeping gene, $\beta$-actin, and were calculated by the $2^{-\Delta \Delta \mathrm{Ct}}$ method.

\section{Western Blot Assay}

The cells were treated with tetrandrine $(0,10 \mu \mathrm{M}$ and 20 $\mu \mathrm{M})$ for $24 \mathrm{~h}$. After washing with PBS, the cells were cleaved by adding $100 \mu \mathrm{L}$ of RIPA and $1 \mu \mathrm{L}$ of PMSF. The lysate was centrifuged by high-speed and lowtemperature centrifugation. Then, the protein concentrations were measured by the BCA method. Equal amounts of proteins were separated by SDS-PAGE and were transferred onto PVDF membranes. The membranes were blocked in the solution with 5\% skimmed milk powder for $2 \mathrm{~h}$. After washing, the membranes were incubated with primary antibodies that were proportionally diluted overnight at $4^{\circ} \mathrm{C}$. Then, the membranes were washed three times with TBST and incubated at room temperature with secondary antibodies for $2 \mathrm{~h}$. Finally, the protein bands were observed with an enhanced ECL reagent and an imaging system (Clinx, Shanghai, China). ImageJ software was used to analyse the results.

\section{Immunofluorescence}

For immunofluorescence staining, the Ishikawa and HEC1-B cells were cultured in 12-well plates with coverslips. After treatment with tetrandrine $(0,10$, and $20 \mu \mathrm{M})$ for $24 \mathrm{~h}$, the cells were fixed with $4 \%$ paraformaldehyde in PBS for $15 \mathrm{~min}$. Next, the cells were permeabilized with $0.3 \%$ Triton $\mathrm{X}-100$ in PBS for 10 min, and blocked with $10 \%$ goat serum in PBS for $1 \mathrm{~h}$. The cells were incubated with p-AKT overnight at $4^{\circ} \mathrm{C}$. After washing with PBS three times, the cells were incubated in the dark with the FITC secondary antibody for $2 \mathrm{~h}$. The nuclei were stained with $5 \mu \mathrm{g} / \mathrm{mL}$ Hoechst 33258 for $5 \mathrm{~min}$. The stained samples were washed with PBS three times. Then, the stained coverslips were visualized and photographed with a fluorescence microscope (Olympus, Tokyo, Japan) at $\times 400$.

\section{Statistical Analysis}

SPSS 17.0 software was used to analyse the data. All data were expressed as the means \pm SD. Student's $t$-test or oneway analysis of variance (ANOVA) was used to analyse the the differences among groups. Values of $\mathrm{P}<0.05$ were considered to be statistically significant.

\section{Results}

\section{Targets of Tetrandrine and EC}

The chemical structure of tetrandrine is shown in Figure 2A. A total of 278 targets associated with tetrandrine were retrieved by removing duplicates. From the TCMSP and DrugBank databases, we obtained 17 and 1 direct target, respectively. Using PharmMapper, we predicted 263 potential targets of tetrandrine. To identify the human genes relevant to EC, the GeneCards, OMIM and DisGeNET databases were used. A total of 728 disease

A<smiles>COc1cc2c3cc1Oc1cc(ccc1C[C@H]1c4cc(cc(OC)c4OC)CCN1C)CCN(C)[C@H]3Cc1ccc(cc1)O2</smiles>

Tetrandrine

(Tet)

B

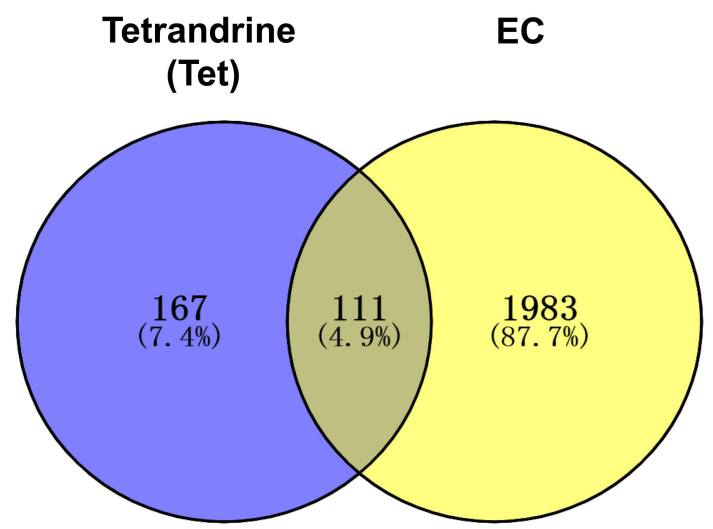

Figure 2 (A) Chemical structure of tetrandrine. (B) Venn diagram of tetrandrine targets and endometrial cancer targets. 
targets were obtained from the GeneCards database by setting the relevance score $\geq 10$. From the OMIM database, 500 targets were related to EC. Moreover, a total of 1148 targets related to $\mathrm{EC}$ were retrieved from the DisGeNET database with a score $\geq 0.8$. A total of 2376 targets were obtained from the three databases. However, there were 282 duplicates among the 2376 targets. After the duplicated studies were removed, 2094 targets remained. Subsequently, the targets of tetrandrine and EC were placed into Venny 2.1 to find 111 common targets. The common targets are shown Figure 2B.

\section{PPI Network Analysis}

The 111 common target genes were input into the STRING database to explore their protein-protein interactions, and a confidence score $>0.9$ was selected. As shown in Figure 3A, there were 111 nodes and 392 edges in the interaction network. The STRING data file was imported into Cytoscape for further analysis of the interaction relationships. According to degree $\geq 20$, betweenness centrality $\geq 0.04$ and closeness centrality $\geq 0.4886,7$ genes were identified that comprised the central genes, which included PIK3R1, AKT1, SCR, HRAS, TP53, MAPK8, and MAPK14 (Figure 3B). Afterwards, the "drug-targetdisease" network was described in Figure 3C by Cytoscape3.7.2.

\section{GO and Pathway Analysis}

To further investigate the potential mechanisms of tetrandrine acting on endometrial cancer, GO function and KEGG enrichment analysis were performed with WebGestalt. GO enrichment analysis revealed that the predicted therapeutic targets were mainly associated with 3 biological processes: metabolic processes, responses to stimulus, and biological regulation; 3 cellular components: nucleus, cytosol, membrane enclosed lumen; and 2 molecular functions: protein binding and ion binding (Figure 4A). As revealed in Figure 4B, KEGG pathway analysis showed that the 111 targets were strongly correlated with 10 pathways, including prostate cancer, pancreatic cancer, and the PI3K-Akt signalling pathway. Moreover, the KEGG map showed that the common target genes were mainly distributed in the cancer pathway. The PI3K-Akt signalling pathway (Figure 4C) is a crucial pathway that includes 32 potential therapeutic targets. It is well known that the PI3K/Akt pathway is a critical pathway that is associated with cancer. These findings suggested that tetrandrine may protect against endometrial cancer through the PI3K-Akt pathway. Based on the results of the network analysis, in vitro experiments were designed to verify our prediction.

\section{Docking Results Analysis}

In this study, Akt1 protein and tetrandrine were selected for molecular docking analysis. AutoDock Vina software output the results in the form of affinity. Affinity can be used to evaluate the affinities between ligands and receptor molecules. Generally, an affinity score <-5.0 kcal/mol indicates that the active component has good binding activity with the target. The docking score of Akt1 protein and tetrandrine was $-8.0 \mathrm{kcal} / \mathrm{mol}$. The results showed that tetrandrine had good binding activity to Akt1 (Figure 5).

\section{Tetrandrine Inhibited Endometrial Cancer Cell Proliferation}

The CCK-8 assay was carried out to evaluate the cytotoxic effect of tetrandrine on endometrial cancer cell lines. Ishikawa and HEC-1-B cells were treated with different tetrandrine concentrations $(0,5,10,20,40$, and $80 \mu \mathrm{M})$ for 24,48 , and $72 \mathrm{~h}$. The viability rate of the non-treatment cells ( $0 \mu \mathrm{M}$ tetrandrine) was defined as $100 \%$, and the viability rate of cells from all other concentrations of tetrandrine was calculated separately from that of the control group (0 $\mu \mathrm{M}$ tetrandrine). As shown in Figure 6, tetrandrine reduced the viability of both cell lines in dose- and time-dependent manners.

\section{Tetrandrine Induced DNA Fragmentation and Apoptosis in Endometrial Cancer Cells}

As the microscopy images in Figure 7A show, the blue staining intensities increased and the number of cells was reduced with higher tetrandrine concentrations. These findings indicated that tetrandrine induced nuclear chromatin condensation in the Ishikawa and HEC-1-B cells. To investigate the proapoptotic effect of tetrandrine, we used a flow cytometry assay with Annexin V-FITC/PI double staining. The group that was treated with $0 \mu \mathrm{M}$ tetrandrine served as the control. As shown in Figure 7B and $\mathrm{C}$, after treatment with tetrandrine (10 and $20 \mu \mathrm{M})$ for $24 \mathrm{~h}$, the apoptotic rates of Ishikawa cells were 26.08 \pm 3.45 , and $37.74 \pm 3.47 \%$, respectively, which were significantly higher than that of the control group (7.40 $\pm 1.70 \%, \mathrm{P}<0.05)$. For the HEC-1B cells, the apoptotic rates of cells that were treated with tetrandrine $(0,10$, 
A

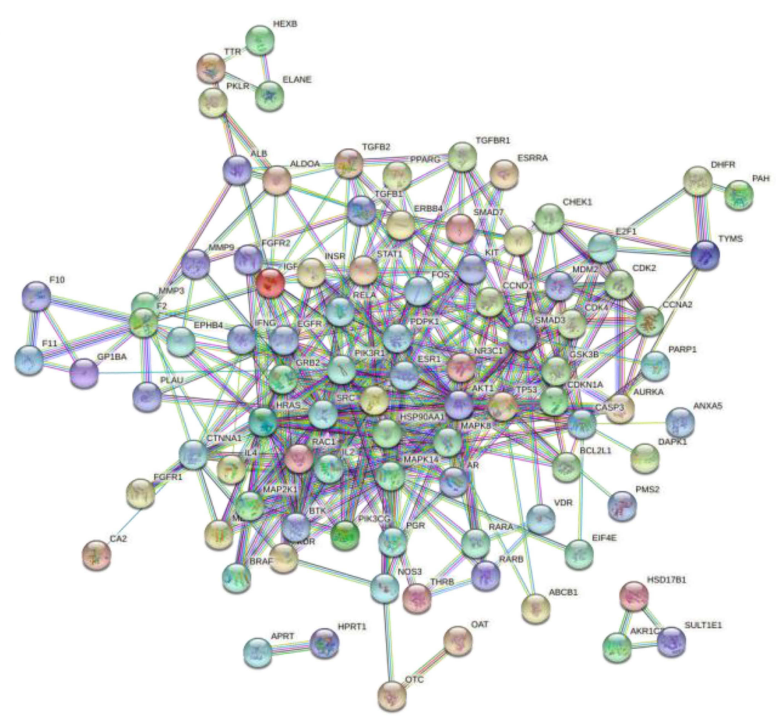

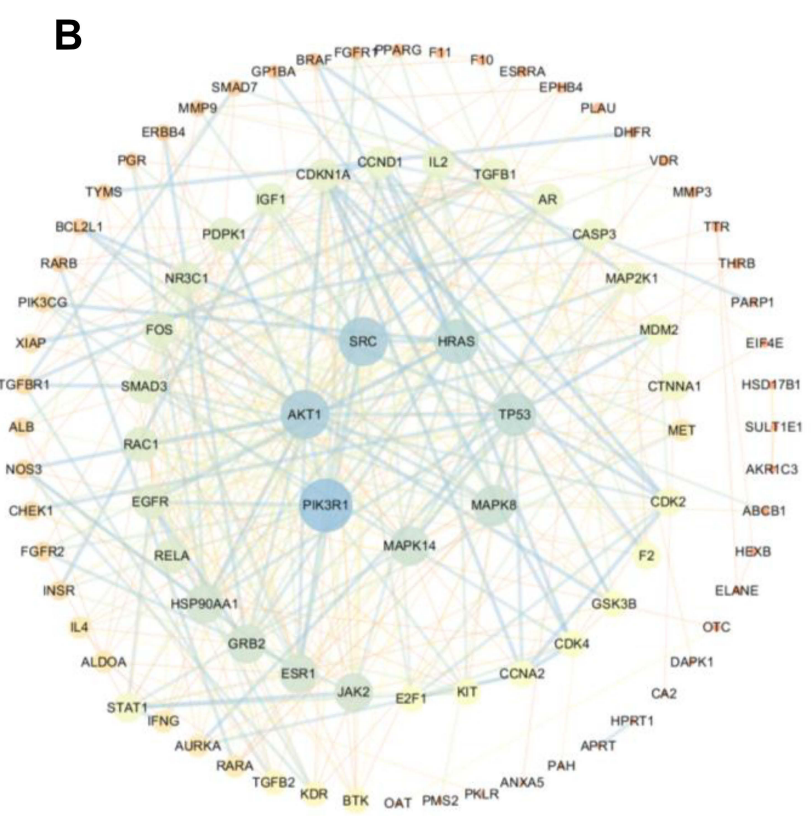

C

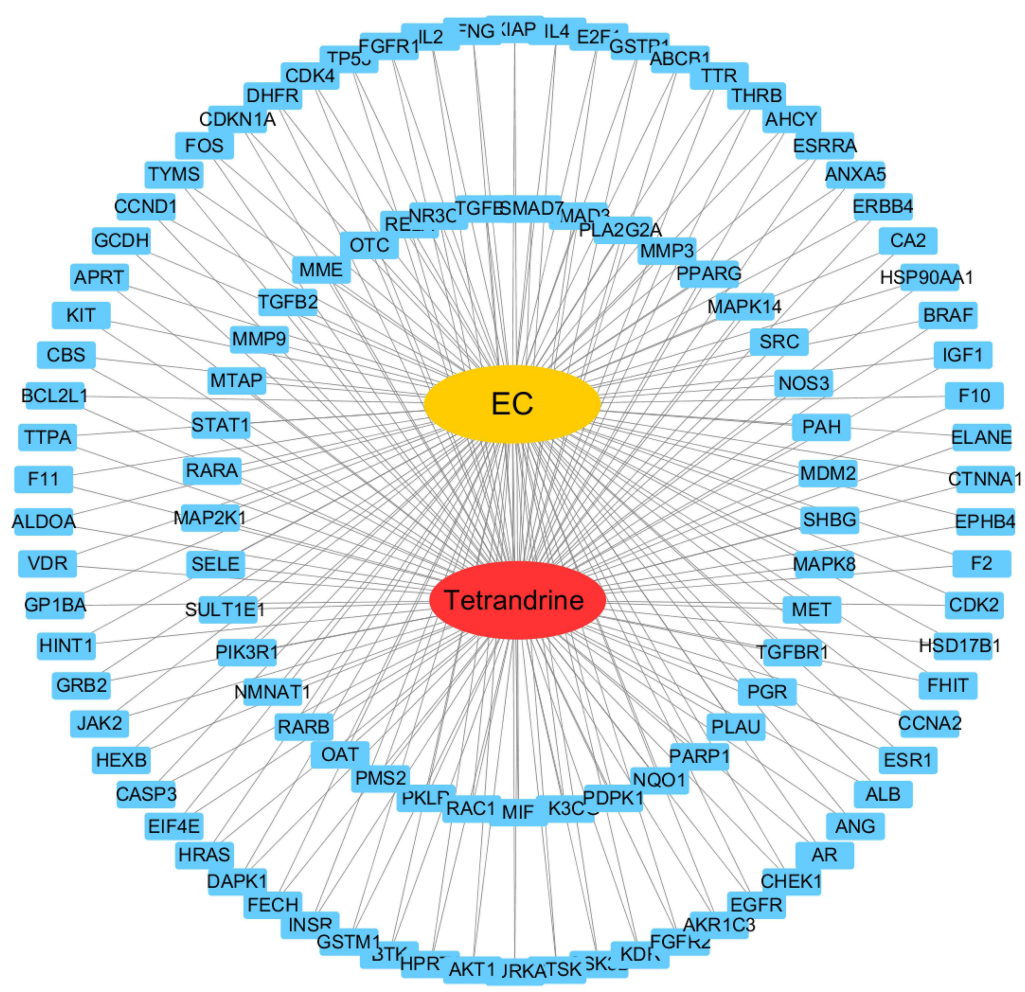

Figure 3 (A) PPI network with the common targets of tetrandrine and endometrial cancer. (B) The 7 core targets in endometrial cancer. (C) "tetrandrine-target-EC" network. Red indicates tetrandrine; Blue indicates drug-disease intersection proteins; Yellow indicates EC.

and $20 \mu \mathrm{M})$ were $6.59 \pm 1.44,25.97 \pm 2.35$, and 42.67 $\pm 2.39 \%$, respectively. These data suggested tetrandrine induced apoptosis in Ishikawa and HEC-1-B cells in a dose-dependent manner.

\section{Effect of Tetrandrine on the mRNA and Protein Expressions of $\mathrm{Bax}$ and $\mathrm{Bcl}-2$}

The qRT-PCR assay showed that the mRNA levels of bcl2 were decreased and that the mRNA levels of bax 
A

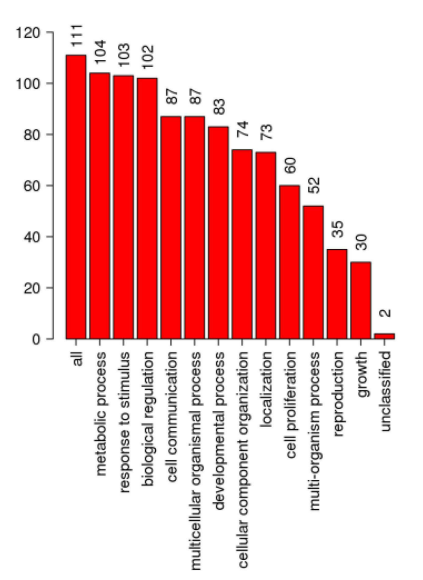

Bar chart of Cellular Component categories

Bar chart of Molecular Function categories
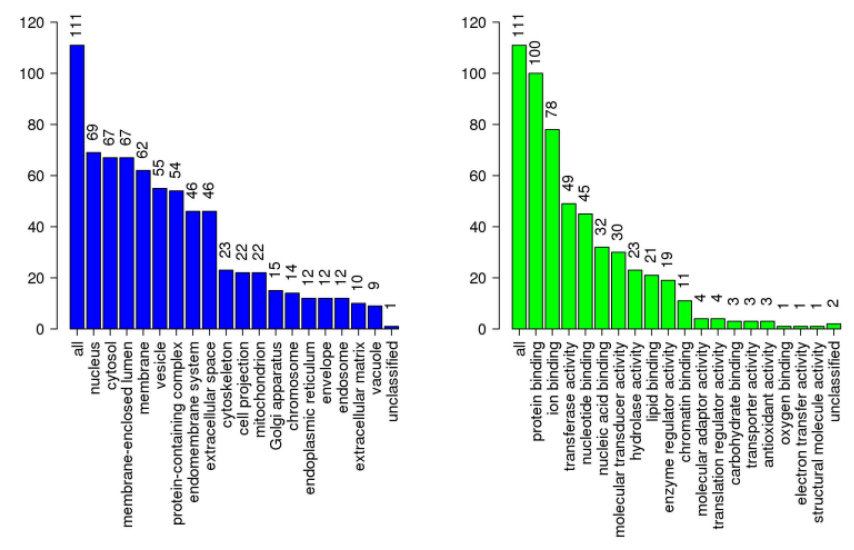

B $\square$ mpr $\leq 005 \square$ m
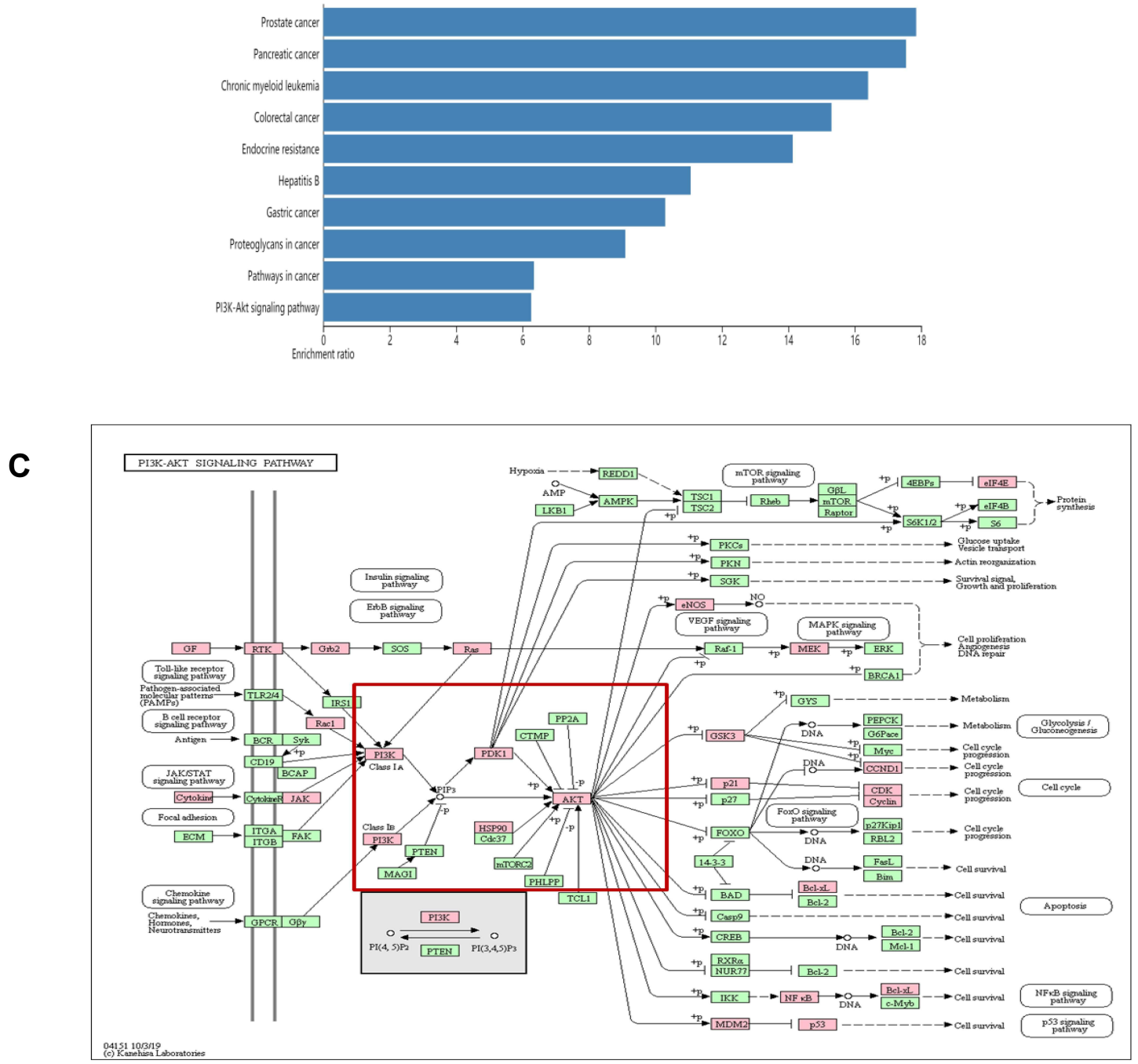

Figure 4 (A) GO map of putative target genes. (B) Kegg pathway analysis of putative target genes. (C) Kegg map of PI3K/Akt signaling pathway. 
A

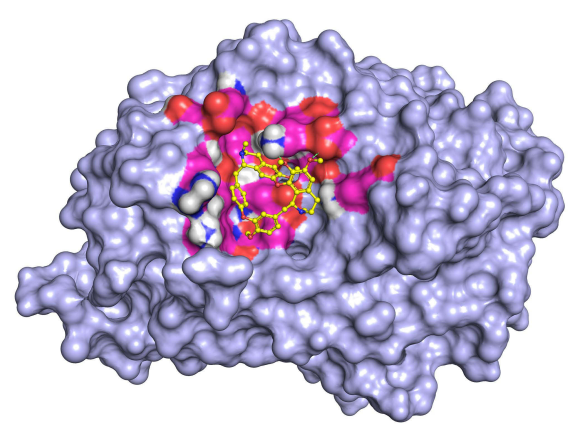

B

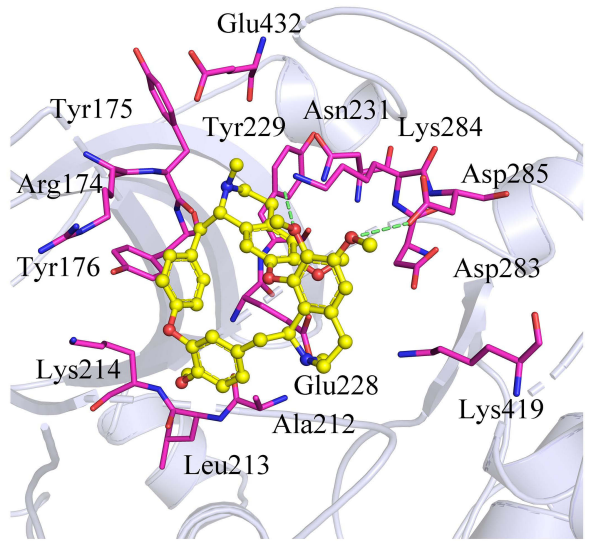

Figure 5 Molecular docking simulation of tetrandrine to Aktl. (A) Holistic Big picture. (B) Local picture.
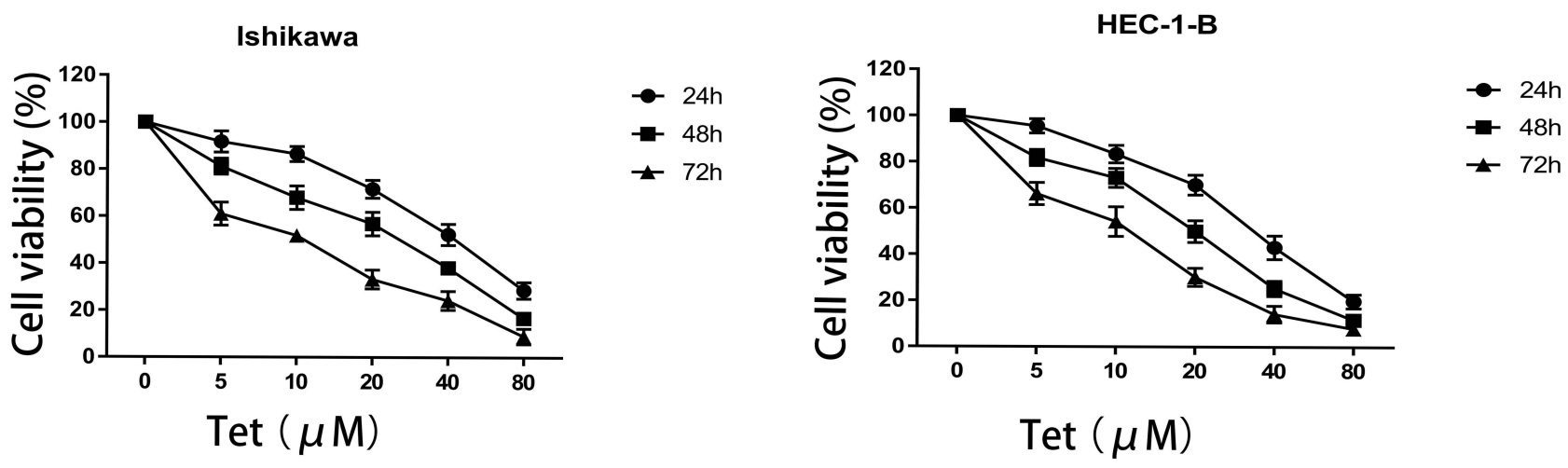

Figure 6 Effect of tetrandrine on the proliferation of Ishikawa and HEC-I-B cells. Both cell lines were treated with tetrandrine $(0,5,10,20,40$, and $80 \mu \mathrm{M})$ for 24,48 , and $72 \mathrm{~h}$. The cell viabilities were measured by CCK-8 assay.

markedly increased, as the tetrandrine concentration increased in Ishikawa and HEC-1-B cells (Figure 8A). Then, the protein levels of Bcl-2 and Bax were evaluated by Western blot analysis. As shown in Figure $8 \mathrm{~B}$ and $\mathrm{C}$, the expression of Bcl-2 was downregulated by tetrandrine $(\mathrm{P}<0.05)$, whereas the protein expression of Bax was upregulated $(\mathrm{P}<0.05)$.

\section{Effect of Tetrandrine on the PI3K/Akt Pathway in Endometrial Cancer Cells}

Western blot assay and qRT-PCR were carried out to investigate the mechanism of tetrandrine regulated cell proliferation and apoptosis. $\mathrm{PI} 3 \mathrm{~K} / \mathrm{Akt}$ signalling is commonly disrupted in human cancers, with Akt being a central component of the pathway, which influences multiple processes that are directly involved in tumourigenesis. ${ }^{20}$ Furthermore, our results predicted that PI3K-Akt signalling may be a key pathway by which tetrandrine protects against endometrial cancer. Hence, we explored the effect of tetrandrine on Akt expression. The qRT-PCR results showed that there were no significant differences in the mRNA levels of Akt during tetrandrine treatment (Figure 9A). However, as shown in Figure $9 \mathrm{~B}$ and $\mathrm{C}$, tetrandrine remarkably downregulated the protein expression ratios of $\mathrm{p}$-Akt/Akt in a dosedependent manner in both Ishikawa and HEC-1-B cells. These data indicated that the inhibition of proliferation and apoptosis induced by tetrandrine may be associated with the downregulation of the PI3K/Akt signalling pathway. To further examine whether tetrandrine downregulates p-Akt, we surveyed the locations of p-Akt in cells with an immunofluorescence assay. As shown in Figure 9D and E, the relative fluorescence intensities of $\mathrm{p}$-Akt in the tetrandrine treatment group were lower than those in the control group (0 $\mu \mathrm{M}$ tetrandrine), which agreed with the Western blot assay results. These data indicated that the inhibition of proliferation and apoptosis induced by tetrandrine may be associated with downregulation of the PI3K/Akt signalling pathway. 
A
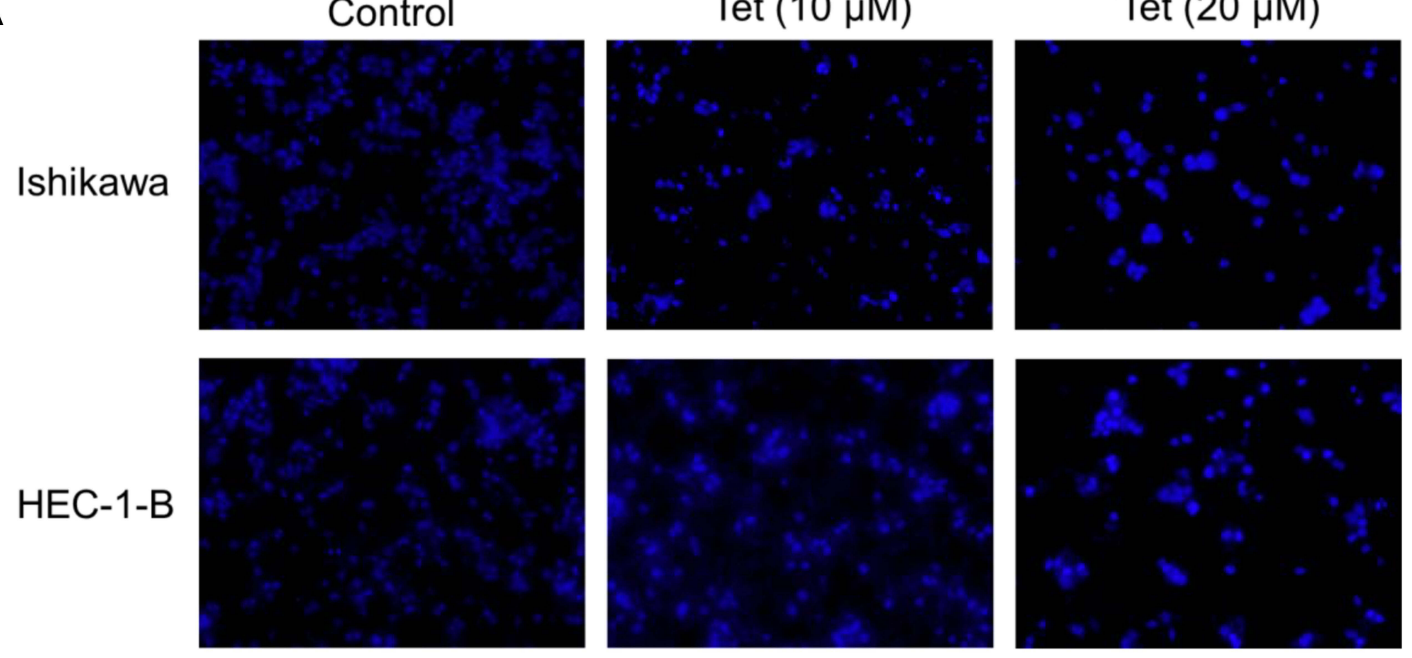

Control

B

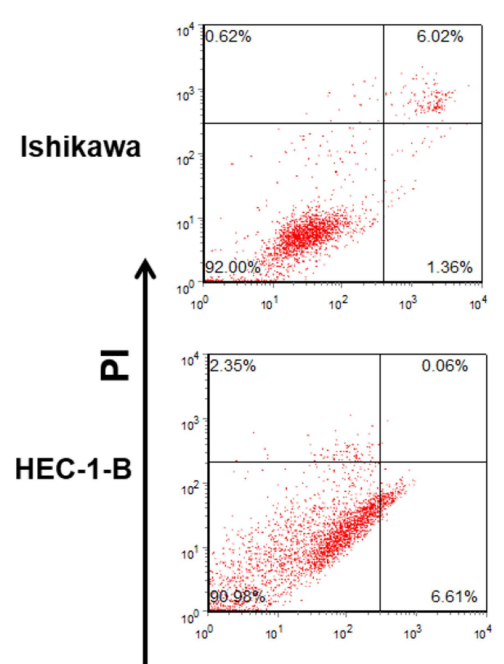

Tet $(10 \mu \mathrm{M})$
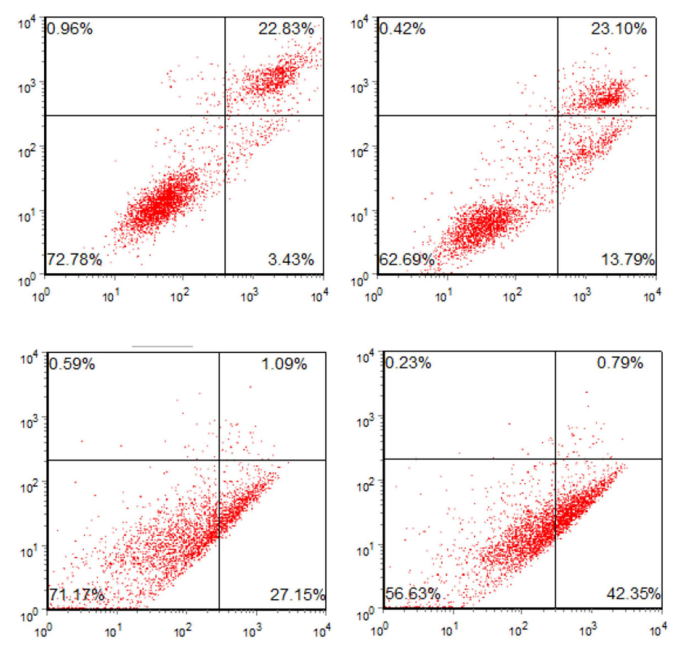

Annexin V

C

Ishikawa

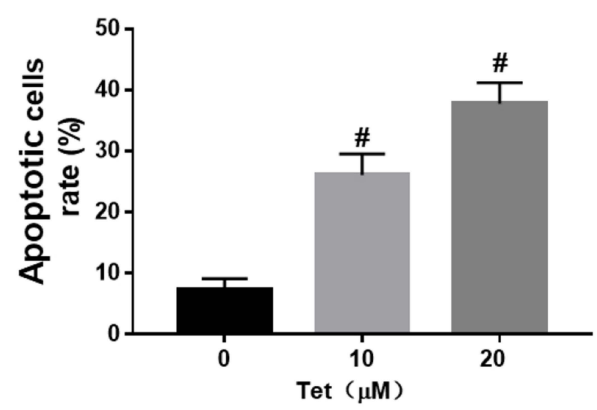

HEC-1-B

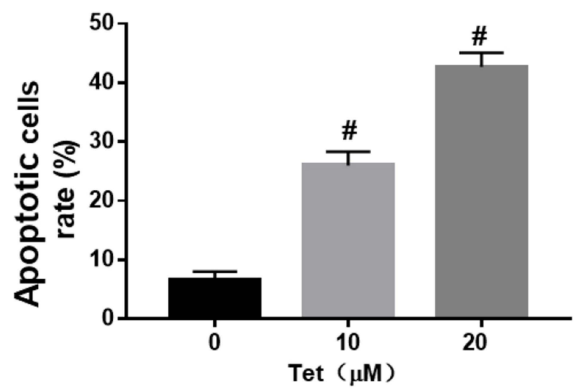

Figure 7 Tetrandrine induced DNA fragmentation and apoptosis in endometrial cancer cells. The Ishikawa and HEC-I-B cells were treated with 0 , I0 or $20 \mu \mathrm{M}$ tetrandrine for $24 \mathrm{~h}$. (A) Hoechst 33258 staining showed typical apoptotic morphology changes (scale bars: $100 \mu \mathrm{m}$ ). (B) The cell apoptosis was assessed by Annexin V- FITC/PI staining. (C) Histogram analysis showed the percentage of apoptotic. Data were presented as means \pm SD ( $n=3),{ }^{*} P<0.05$. 
A

Ishikawa

HEC-1-B

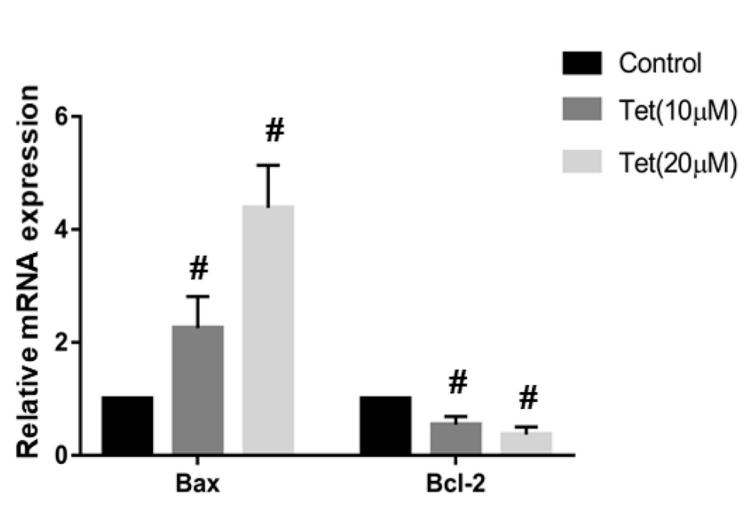

B

Ishikawa

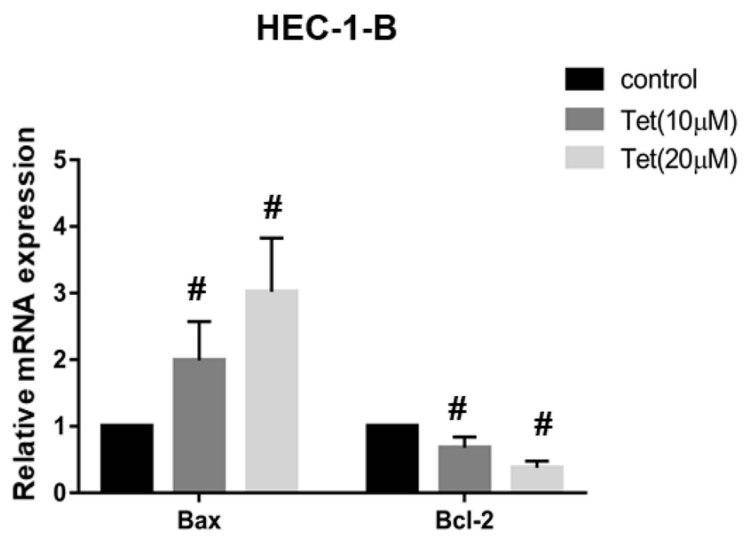

HEC-1-B
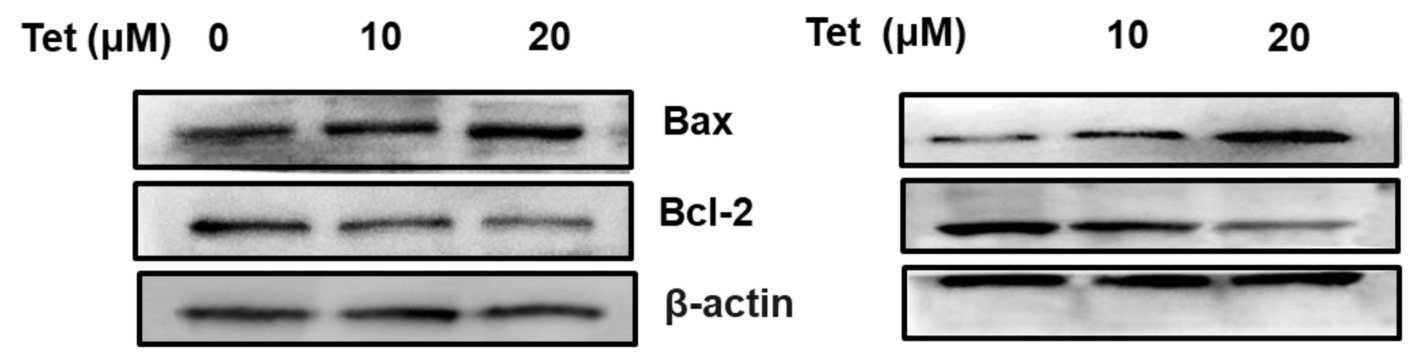

Bax

$\mathrm{Bcl}-2$
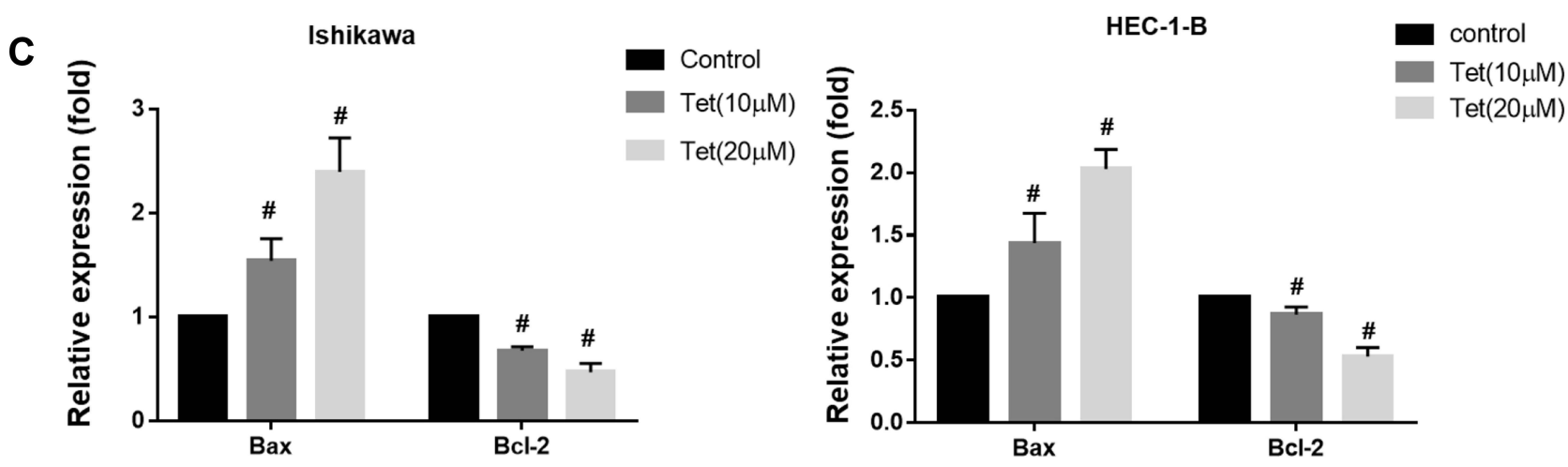

Figure 8 Effect of tetrandrine on the mRNA and protein expressions of Bax and Bcl-2. The Ishikawa and HEC-I-B cells were treated with 0 , 10 or $20 \mu \mathrm{M}$ tetrandrine for 24 h. (A) The mRNA expression levels of Bax and Bcl-2 were measured by qRT-PCR. (B) The protein expression levels of Bax and Bcl-2 were detected by Western blotting. (C) The relative protein expression of $B a x$ and $B c l-2$ were normalized to $\beta$-actin. Data were presented as means $\pm S D(n=3)$, ${ }^{\#} P<0.05$.

\section{Discussion}

In recent years, with changes in human habits and diets, endometrial cancer has shown increasing incidence rates and younger onsets. Over the past 20 years, its incidence has risen by more than $50 \%{ }^{21}$ To date, there is no welltolerated and effective treatment for advanced and recurrent endometrial cancer. TCMs are a significant source for cancer treatments. Recently, the promise of TCM in cancer treatments has been increasingly recognized, and TCM is one of the most common complementary therapies for chemotherapy, surgery, and radiotherapy that is used worldwide, especially in Asia. ${ }^{22}$ Network pharmacology provides an appropriate approach for drug-target exploration and modern TCM research. ${ }^{23}$ Previous studies have verified that tetrandrine can inhibit cancer cell growth and progression in multiple types of cancers. To date, studies of tetrandrine against EC have not been reported. Therefore, in this study, the key mechanism of tetrandrine against endometrial cancer was investigated by using a combination of network pharmacology prediction and 
A

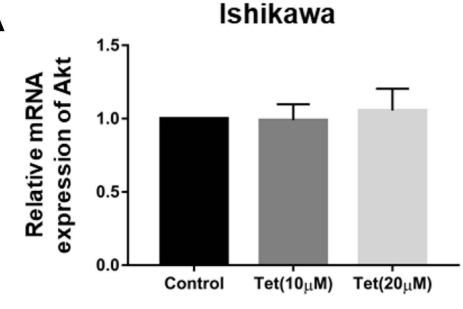

B

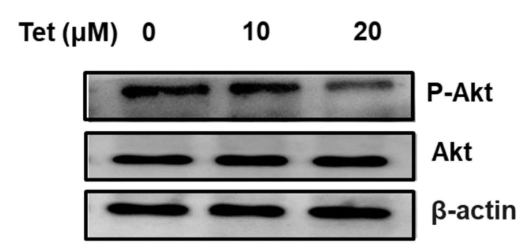

C

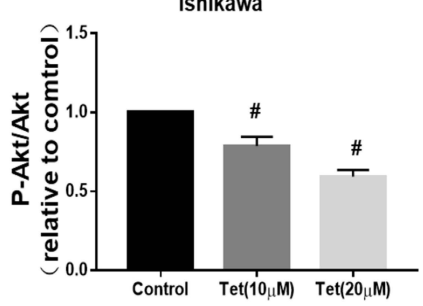

HEC-1-B

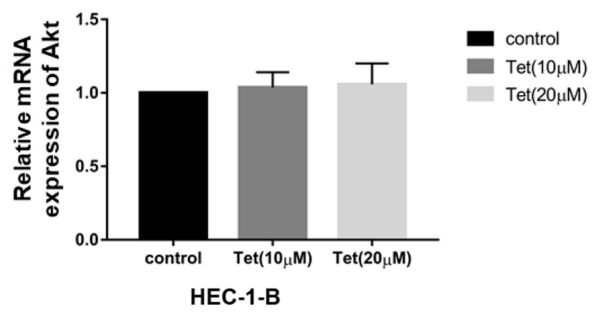

Tet $(\mu M) 0 \quad 10 \quad 20$

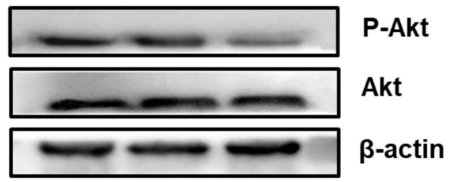

HEC-1-B

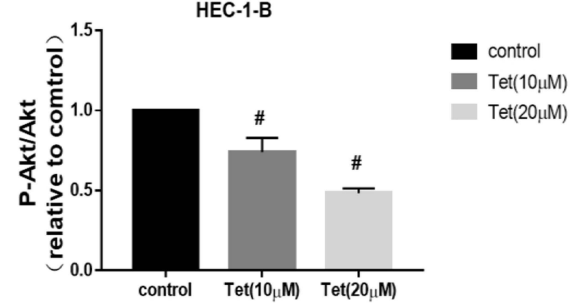

D
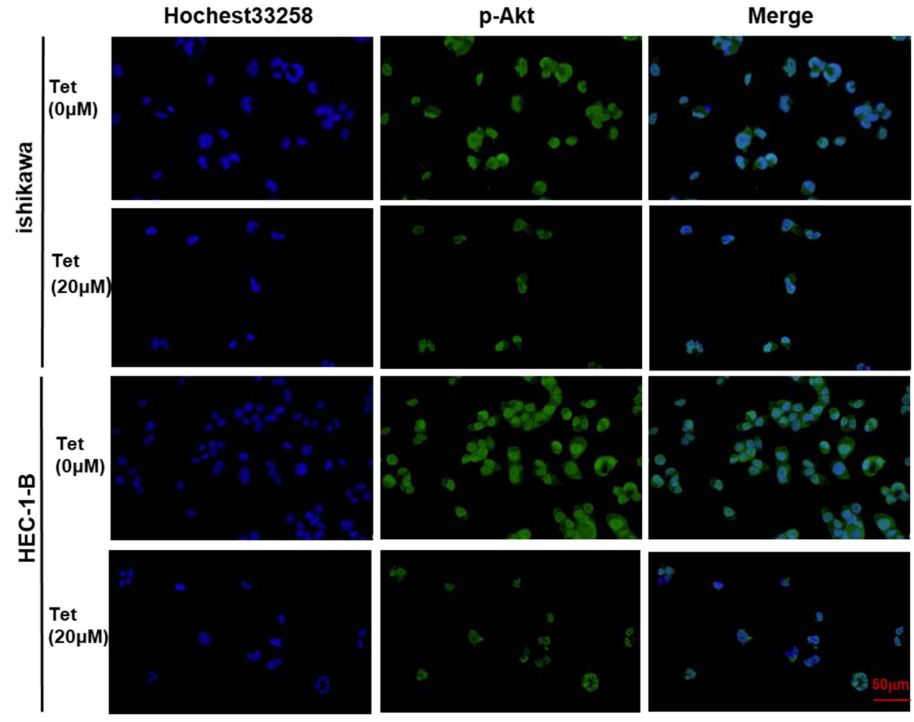

E

ishikawa

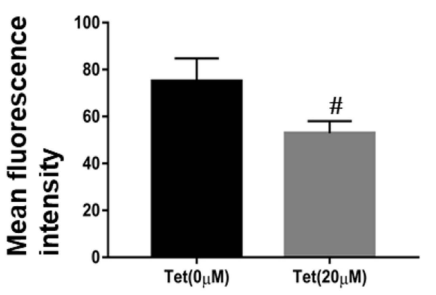

HEC-1-B

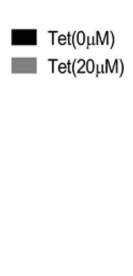

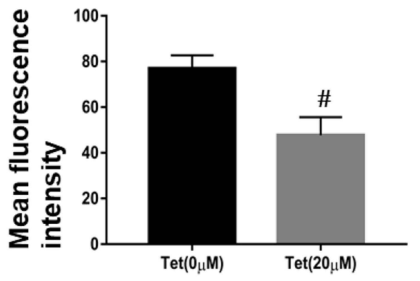

Figure 9 Tetrandrine repressed the PI3K/Akt signalling pathway in endometrial cancer cells. The Ishikawa and HEC-I-B cells were treated with 0 , I0 or $20 \mu \mathrm{M}$ tetrandrine for 24 h. (A) The mRNA expression levels of Akt were measured by qRT-PCR. (B) The protein expression levels of p-Akt and Akt were detected by Western blotting. (C) The ratio of $p$-Akt/Akt was relative to control group $\left(0 \mu \mathrm{M}\right.$ tetrandrine). ${ }^{\#} \mathrm{P}<0.05$. (D) Immunofluorescence staining of $\mathrm{p}$-Akt was observed by a fluorescence microscope (scale bars: $50 \mu \mathrm{m})$. (E) Quantitative analysis of $\mathrm{p}$-Akt immunofluorescence. Data were presented as means \pm SD $(\mathrm{n}=3),{ }^{\#} \mathrm{P}<0.05$. 
experimental validation. Through the use of publicly available databases, we obtained 111 potential targets between tetrandrine and EC. Then, the PPIs of the common targets were constructed, and the key gene targets were obtained. We constructed a drug-target-disease network to describe these interactions. To further investigate the potential therapeutic targets, we performed GO function and KEGG pathway enrichment with WebGestalt. GO enrichment analysis indicated that these targets were associated with metabolic processes, responses to stimulus, and biological regulation. The KEGG pathway analysis showed that the common target genes were mainly distributed in the PI3K/ Akt signalling pathway. Moreover, the molecular docking results showed that tetrandrine exhibited good binding activity to Akt1, which was one of the key targets.

Additional in vitro experiments were performed to verify the predicted molecular mechanisms of tetrandrine against EC. In our study, the effects of tetrandrine on proliferation were investigated by a CCK-8 assay. The experiments revealed that tetrandrine inhibited endometrial cancer cells in a time- and dose-dependent manner. Flow cytometric analysis indicated that tetrandrine induced apoptosis of endometrial cancer cells with a dose-dependent pattern.

Apoptosis, a form of programmed cell death, is responsible for tumour development and progression. In cancer, deregulated apoptotic signaling, particularly the activation of antiapoptotic systems, allows cancer cells to escape this program, which leads to uncontrolled proliferation and tumour survival, therapeutic resistance and recurrence of cancer. ${ }^{24}$ The Bcl-2 gene family has a central role in regulating programmed cell death by controlling pro-apoptotic and anti-apoptotic intracellular signals. ${ }^{25}$ In the Bcl-2 family, Bax is a proapoptotic member, while Bcl-2 is an antiapoptotic member. When unbalanced, the Bcl-2 family can act as a barrier to apoptosis and facilitate tumour development and resistance to cancer therapy. ${ }^{26}$ In our study, the results revealed that the mRNA and protein expression levels of Bcl-2 were significantly downregulated, while the expression levels of Bax were upregulated in the groups treated with tetrandrine. Therefore, apoptosis that was induced by tetrandrine in endometrial cancer cells was associated with Bcl-2 family proteins.

The PI3K/ Akt pathway is one of the most frequently dysregulated pathways in cancer that regulates cell proliferation, growth, cell size, metabolism, and motility. ${ }^{27,28}$ Akt participates in the key role of the pathway, and the abnormal overexpression or activation of Akt has been observed in many cancers, including ovarian, lung, and pancreatic cancers. ${ }^{29}$ In addition, overactivation of the PI3K/ Akt pathway has recently been implicated in endometrial cancer pathogenesis, and due to this, inhibition of the PI3K/ Akt pathway is of therapeutic interest. ${ }^{30}$ Therefore, to verify the prediction that we obtained from biological data mining, we determined the expressions of Akt and p-Akt by using Western blot assays. The experimental results showed that the expression ratios of p-Akt/Akt significantly decreased in the tetrandrine groups. Furthermore, immunofluorescence assays confirmed that the relative fluorescence intensities of p-Akt decreased as the tetrandrine dose increased. Taken together, these results revealed that tetrandrine exerted tumour suppressive effects on EC by regulating the PI3K/Akt signalling pathway. However, this study still has limitations. First, the mechanism of tetrandrine against endometrial cancer is complicated and involves multiple pathways. We need to further explore other mechanisms. In addition, it is necessary to conduct additional in vivo experiments to further confirm the results.

In conclusion, we revealed that the PI3K/Akt signalling pathway is a promising target that is relevant to the mechanism of tetrandrine against endometrial cancer based on network pharmacology approaches. In vitro experiments confirmed that tetrandrine suppresses the proliferation and induces the apoptosis in endometrial cancer cells via the PI3K/Akt pathway. These results suggest that tetrandrine is a promising and effective multitargeted medication for endometrial cancer.

\section{Acknowledgments}

This study was supported by the Zibo City Key R\&D Program (2018kj010120, 2018kj010010).The authors would like to acknowledge the help of Mr. Jun Li, who provided the Ishikawa cells. The experiments were performed at the laboratory of the Translational Medical Center and Shandong University of Technology.

\section{Disclosure}

The authors declare no conflicts of interest.

\section{References}

1. Siegel RL, Miller KD, Fuchs HE, Jemal A. Cancer Statistics, 2021. CA Cancer J Clin. 2021;71(1):7-33. doi:10.3322/caac.21654

2. Lu KH, Broaddus RR, Longo DL. Endometrial Cancer. $N$ Engl J Med. 2020;383(21):2053-2064. doi:10.1056/NEJMra1514010

3. Eritja N, Yeramian A, Chen BJ, et al. Endometrial carcinoma: specific targeted pathways. Adv Exp Med Biol. 2017;943:149-207.

4. Xiang Y, Guo Z, Zhu P, Chen J, Huang Y. Traditional Chinese medicine as a cancer treatment: modern perspectives of ancient but advanced science. Cancer Med. 2019;8(5):1958-1975. doi:10.1002/ cam4.2108

5. Bhagya NC, Chandrashekar KR. Tetrandrine and cancer - An overview on the molecular approach. Biomed Pharmacother. 2018;97:624-632. doi:10.1016/j.biopha.2017.10.116 
6. Bhagya N, Chandrashekar KR. Tetrandrine-A molecule of wide bioactivity. Phytochemistry. 2016;125:5-13. doi:10.1016/j. phytochem.2016.02.005

7. Zhang H, Xie B, Zhang Z, Sheng X, Zhang S. Tetrandrine suppresses cervical cancer growth by inducing apoptosis in vitro and in vivo. Drug Des Devel Ther. 2019;13:119-127. doi:10.2147/DDDT. S187776

8. Lin YJ, Peng SF, Lin ML, et al. Tetrandrine induces apoptosis of human nasopharyngeal carcinoma NPC-TW 076 cells through reactive oxygen species accompanied by an endoplasmic reticulum stress signaling pathway. Molecules. 2016;21(10):1353. doi:10.3390/ molecules21101353

9. Yu B, Yuan B, Li J, et al. JNK and autophagy independently contributed to cytotoxicity of arsenite combined with tetrandrine via modulating cell cycle progression in human breast cancer cells. Front Pharmacol. 2020;11:1087. doi:10.3389/fphar.2020.01087

10. Wu K, Zhou M, Wu QX, et al. The role of IGFBP-5 in mediating the anti-proliferation effect of tetrandrine in human colon cancer cells. Int J Oncol. 2015;46(3):1205-1213. doi:10.3892/ijo.2014.2800

11. Lien JC, Lin MW, Chang SJ, et al. Tetrandrine induces programmed cell death in human oral cancer CAL 27 cells through the reactive oxygen species production and caspase-dependent pathways and associated with beclin-1-induced cell autophagy. Environ Toxicol. 2017;32(1):329-343. doi:10.1002/tox.22238

12. Kou B, Liu W, He W, et al. Tetrandrine suppresses metastatic phenotype of prostate cancer cells by regulating Akt/mTOR/MMP-9 signaling pathway. Oncol Rep. 2016;35(5):2880-2886. doi:10.3892/ or.2016.4649

13. Poornima P, Kumar JD, Zhao Q, Blunder M, Efferth T. Network pharmacology of cancer: from understanding of complex interactomes to the design of multi-target specific therapeutics from nature. Pharmacol Res. 2016;111:290-302. doi:10.1016/j. phrs.2016.06.018

14. Kibble M, Saarinen N, Tang J, Wennerberg K, Makela S, Aittokallio T. Network pharmacology applications to map the unexplored target space and therapeutic potential of natural products. Nat Prod Rep. 2015;32(8):1249-1266. doi:10.1039/C5NP00005J

15. Yuan H, Ma Q, Cui H, et al. How can synergism of traditional medicines benefit from network pharmacology? Molecules. 2017;22 (7):1135. doi:10.3390/molecules22071135

16. Ru J, Li P, Wang J, et al. TCMSP: a database of systems pharmacology for drug discovery from herbal medicines. J Cheminform. 2014;6 (1):13. doi:10.1186/1758-2946-6-13

17. Liu X, Ouyang S, Yu B, et al. PharmMapper server: a web server for potential drug target identification using pharmacophore mapping approach. Nucleic Acids Res. 2010;38(suppl_2):W609-14. doi:10.1093/nar/gkq300
18. Wang X, Pan C, Gong J, Liu X, Li H. Enhancing the enrichment of pharmacophore-based target prediction for the polypharmacological profiles of drugs. J Chem Inf Model. 2016;56(6):1175-1183. doi:10.1021/acs.jcim.5b00690

19. Wang X, Shen Y, Wang S, et al. PharmMapper 2017 update: a web server for potential drug target identification with a comprehensive target pharmacophore database. Nucleic Acids Res. 2017;45(W1): W356-W60. doi:10.1093/nar/gkx374

20. Brown JS, Banerji U. Maximising the potential of AKT inhibitors as anti-cancer treatments. Pharmacol Ther. 2017;172:101-115. doi:10.1016/j.pharmthera.2016.12.001

21. MacKintosh ML, Crosbie EJ. Prevention strategies in endometrial carcinoma. Curr Oncol Rep. 2018;20(12):101. doi:10.1007/s11912018-0747-1

22. Liu WY, Zhang JW, Yao XQ, et al. Shenmai injection enhances the cytotoxicity of chemotherapeutic drugs against colorectal cancers via improving their subcellular distribution. Acta Pharmacol Sin. 2017;38(2):264-276. doi:10.1038/aps.2016.99

23. Zhang R, Zhu X, Bai H, Ning K. Network pharmacology databases for traditional Chinese medicine: Review and assessment. Front Pharmacol. 2019;10:123. doi:10.3389/fphar.2019.00123

24. Mohammad RM, Muqbil I, Lowe L, et al. Broad targeting of resistance to apoptosis in cancer. Semin Cancer Biol. 2015;35 Suppl:S78S103.

25. Ashkenazi A, Fairbrother WJ, Leverson JD, Souers AJ. From basic apoptosis discoveries to advanced selective BCL-2 family inhibitors. Nat Rev Drug Discov. 2017;16(4):273-284. doi:10.1038/ nrd.2016.253

26. Campbell KJ, Tait SWG. Targeting BCL-2 regulated apoptosis in cancer. Open Biol. 2018;8(5):180002. doi:10.1098/rsob.180002

27. Janku F, Yap TA, Meric-Bernstam F. Targeting the PI3K pathway in cancer: are we making headway? Nat Rev Clin Oncol. 2018;15 (5):273-291. doi:10.1038/nrclinonc.2018.28

28. Alzahrani AS. PI3K/Akt/mTOR inhibitors in cancer: at the bench and bedside. Semin Cancer Biol. 2019;59:125-132. doi:10.1016/j. semcancer.2019.07.009

29. Song M, Bode AM, Dong Z, Lee MH. AKT as a Therapeutic Target for Cancer. Cancer Res. 2019;79(6):1019-1031. doi:10.1158/00085472.CAN-18-2738

30. Slomovitz BM, Coleman RL. The PI3K/AKT/mTOR pathway as a therapeutic target in endometrial cancer. Clin Cancer Res. 2012;18(21):5856-5864. doi:10.1158/1078-0432.CCR-12-0662

\section{Publish your work in this journal}

Drug Design, Development and Therapy is an international, peerreviewed open-access journal that spans the spectrum of drug design and development through to clinical applications. Clinical outcomes, patient safety, and programs for the development and effective, safe, and sustained use of medicines are a feature of the journal, which has also been accepted for indexing on PubMed Central. The manuscript management system is completely online and includes a very quick and fair peer-review system, which is all easy to use. Visit http://www. dovepress.com/testimonials.php to read real quotes from published authors. 